\title{
RFI Mitigation in Radio Astronomy
}

\author{
Willem A. Baan*t \\ ASTRON, Netherlands Institute for Radio Astronomy, Dwingeloo, $N L$ \\ E-mail: baaneastron.nl \\ Department of Mathematics, Physics and Computer Science, Linnaeus University, Växjö, SE
}

\begin{abstract}
This paper presents an overview of methods for mitigating radio frequency interference (RFI) in radio astronomy data. The primary purpose of mitigation is to assist observatories to take useful data outside frequency bands allocated to the Radio Astronomy Service (RAS): mitigation should not be needed within RAS bands. Mitigation methods may be introduced at a variety of points within the data acquisition system. These range from proactive methods to change the local RFI environment by means of regulatory manners, to pre- and post-detection methods, to various precorrelation methods, and to methods applied at correlation and post-correlation. Every method of RFI mitigation results in data loss. A forward look is presented on the implementation of methods to cope with changing interference environments at observatories.
\end{abstract}

RFI mitigation workshop - RFI2010,

March 29-31, 2010

Groningen, the Netherlands

\footnotetext{
*Speaker.

${ }^{\dagger}$ WAB thanks Albert-Jan Boonstra, Peter Fridman and Murray Lewis for valuable discussions about this paper.
} 


\section{Introduction - Objectives}

Interference mitigation in radio astronomy is aimed at reducing or removing the impact of signals from active services in bands outside those allocated to the Radio Astronomy Service (RAS). These efforts should not be aimed at unwanted signals within an RAS band, nor should the ability to mitigate interference serve as an excuse for discounting the presence of unwanted emissions, as interference (almost) always leads to both data loss and to a loss of data quality. Data loss inside allocated RAS bands poses a regulatory problem but there is no recourse for data loss on bands not allocated to the RAS. Indeed the primary benefit from applying a mitigation method is often to reduce the impact of RFI on unaffected data. Since data loss is avoided by prevention, prevention is much better than mitigation. Otherwise the earlier an interference problem is dealt with in the processing chain the better. Early intervention results in less damage to the data, lower downstream costs, and less system complexity. Interference mitigation is possible when there is a significant interference-to-noise ratio (INR). Weak RFI is therefore best addressed later in the processing chain, after integrating the data to increase its signal-to-noise ratio (SNR).

As one passive service among many active services, the RAS is one of the first to suffer from the prevailing philosophy that spectrum efficiency is only enhanced by accommodating more active spectrum users. The ITU standards for threshold levels of permitted spurious and out-of-band (i.e. unwanted) emissions are often insufficient for the protection of active services from each other, and these levels certainly do not suffice to protect the passive services. Rather than concentrating on RFI prevention, it is generally easier (and more cost effective) for spectrum managers to solve interference issues as they arise. However, RAS observations are very sensitive to RFI, so its interference issues are more difficult to solve in this fashion. In addition, current RAS systems operate with increasingly large bandpasses and ever-higher system sensitivity. As a result observed bands often cover bands allocated to active services, where the RAS has no protection status. RFI mitigation techniques are then essential for operating in non-allocated and non-protected bands.

A new threat encountered by the RAS in operating over wider bandwidths comes from the recent introduction of low-power, broadband, spread-spectrum devices that are both unlicensed and mass-produced. Since their adopted spectrum masks are rather inadequate, creative mitigation solutions are required to enable optimal RAS operations. A second threat is dynamic spectrum access (DSA) that allows systems to operate in spectrum slots that are unused for a period of time. While cognitive radio devices are currently designed to operate in bands allocated to particular services, such devices will increasingly fill the spectrum in a new form of spectrum sharing. Of course, RAS broadband operation depends on the same DSA principle as the new cognitive radio devices being introduced into the market place.

Earlier reviews of methods of RFI Mitigation for radio astronomy have been presented by Fridman \& Baan [1], ITU-R RA.2126 [2], and Kesteven [3], and there are reviews of a previous conference by Ellingson [4] and Briggs [5].

\section{Signatures of RFI Sources and their Impact}

The actual impact of interference on data obtained with radio astronomy telescopes depends on a number of factors, the first of which being the type of radio telescope used. Single-dish 
telescopes are the most vulnerable as they have little directional discrimination against incoming RFI, which results in the astronomical and RFI signals being coherently added. This contrasts with the sensitivity to RFI of telescopes incorporated into connected arrays for interferometry, where RFI partly enters the system incoherently. In these configurations the extended baselines serve as a spatial filter that discriminates against RFI. Every interferometric system using 'fringe stopping' techniques has a baseline-dependent capacity for the rejection of RFI, the more so the longer the baseline [6]. Nevertheless, the calibration of each station in an array is still affected by its local RFI.

The second factor depends on the type of observation. While continuum measurements can sacrifice certain time slots or frequency bands within the time-frequency diagram of an observation, a spectral line observation is damaged whenever the frequency and width of the RFI directly affects the astronomical signal.

Thirdly, the impact on the data depends on the type of RFI. Is the RFI time-variable (impulsive), narrow-band or wide-band, and/or is there a superposition of RFI patterns? Direct (and indirect) transmissions from terrestrial networks, that follow population density and the affluence of the local community, give a persistent signal background. Satellites and aeronautical services may cause both steady and transient RFI components. The location of an observatory relative to aeronautical traffic lanes may ensure the presence of their direct emissions and the reflections of terrestrial signals, such as those from airport radars. Most RFI results from side-lobe coupling with active services, though destructive main-beam coupling can arise from satellites and from aeronautical services, as can happen for instance from the Cloudsat satellite [7] at $94 \mathrm{GHz}$. In addition to external RFI, there is also the prospect of gratuitous, observatory-generated RFI from on site computing and electronics: these sources can be identified and should be adequately shielded.

Fourthly, time variability of RFI may particularly impact time-critical astronomical observations. Variable, non-repetitive RFI occurring during studies of transients and pulsars may destroy critical observations that are unique and cannot be repeated. It may affect the time sequencing of pulses, and may also affect the time-critical calibration of data.

Finally, the RFI environment has changed rapidly over recent years, as new service applications are introduced and others phased out. The introduction of broadband, spread-spectrum applications for broadcasting and communication, as well as of unlicensed devices, that take away the peaked, high-power signals of yore by replacing them with broader signals of lower power, generate signals that are not easy to remove from RAS data. The intensification of spectrum use and of dynamic spectrum access will increassingly change the character of the RFI environment, and will increasingly require the radio astronomy community to adjust its approach to reducing the impact of RFI on its data.

\section{RFI Mitigation Methodologies - layers of mitigation}

A number of RFI mitigation methods may be used at different stages in the data acquisition process. The first and most powerful method is to control incoming RFI signals before they enter the receiver, by modifying the local and regional RFI environment. As a second layer, pre-detection methods may be applied in the receiver system itself, possibly in connection with the data-taking backend. A third layer, consisting of digital excision and RFI removal methods, may be used 
before correlation. With the advent of software (SW) correlation, these digital methods may also be incorporated into the correlation process. A fourth possible layer would invoke the application of digital methods after correlation and after data integration or data buffering. Finally, a fifth mitigation layer consists of manipulating (excising and flagging) the collected astronomical data to eliminate the effects of known and unknown sources of RFI.

The performance of all of these methods depends on the INR, i.e. on the relative strength of the RFI or on the ratio of system-noise variance to RFI variance. Most methods are only effective when RFI is clearly detectable within the data, and can only remove its effects down to the instantaneous noise level. A figure of merit for these methods is the processing gain after the RFI suppression or reduction given by the ratio of the SNR(after) to SNR(before).

The quality of the performance of any method depends on the required level of suppression, since each layer of mitigation has its own limitations. The occupied bandwidth of an astronomical signal relative to that of the RFI plays a role here. It is important that loss of the signal-of-interest (SOI) is considered while judging the results of mitigation, as this varies with each method. Indeed a layering of several methods has a cumulative effect, as each subsequent layer of mitigation operates on the remnant of any RFI signal remaining from prior processing. In addition, each applied method can introduce a measure of toxicity, which is the damage done to data that adds incremental degradation to the data quality. The total damage done to data, as a measure of the data loss resulting from (subsequent) mitigation processing, is quantified by the ratio of the SNR(after processing) to the SNR(in the absence of RFI).

\section{Pro-active methods - changing the RFI environment}

Coordination with active users and recourse to national and international regulations may reduce the occurrence of RFI at a radio astronomy station, and may reduce its impact on observations. Improving and strengthening the regulatory framework at national, regional, and international levels plays an important role in protecting passive use of the spectrum: resources in support of this approach are to be found in the Handbook on Radio Astronomy [8], in ITU-R recommendations such as ITU-R RA.769 and RA.1513 [9], and in the CRAF Handbook [10]. Coordination zones and radio quiet zones may be used to modify the RFI environment that would be generated by terrestrial sources of interference at a telescope. Many observatories have local and national regulations that prevent the installation of transmitters in the immediate proximity (within 2-6 kilometers) of an observatory. Large-scale coordination and quiet zones have been implemented for a few sites, such as the National Radio Quiet Zone around Green Bank [11] and the Puerto Rico Coordination Zone around the Arecibo Observatory [12]. The environments for new telescopes, such as ALMA in Chile and the two prospective sites for the Square Kilometer Array, are being regulated by forward-looking, national regulations to enable the most sensitive observations to be undertaken from them.

Because it is better to solve potential RFI issues before implementation, it is important to identify both existing and prospective new transmitters that may affect the portions of the radio spectrum of interest to an observatory, by keeping up with changes in local licensing and by recognizing trends in spectrum use. Spectrum monitoring may be used to identify trends, to identify 
'spectrum neighbors', and to get to 'know thine enemy'. Spectrum monitoring is also a tool to determine the percentage of data loss caused by interference in the passive bands.

Experience shows that observatories are often themselves significant sources of RFI. Computing hardware and electronic installations in buildings can generate harmonic and broadband emissions that enter a telescope's detection system. Identification and elimination of these sources is a high priority for every observatory. RFI-tight cabinets and Faraday cages to accommodate electronics and computing equipment, as well as the reduction of human activity (remote control rooms) and a restriction on local computer use, help to make an observatory 'radio-quiet'. This to-do list is part of the necessary preparations for an observatory to conduct its most sensitive observations [13].

\section{Pre-detection \& post-detection}

A standard method for excising RFI in the frequency domain is to install a bandpass or high/low pass filter in a receiver, which results in an insertion loss and substantially raises the system temperature at frequencies close to a band-edge. Super-conducting filter technology can significantly decrease the impact of such filters. Filtering of RAS bands serves to prevent damage due to strong signals outside the bands. Filtering also results in data loss for continuum observations, while it is often essential to enable spectral line observations when RFI occurs at a critical frequency within a receiver's passband.

Blanking or stopping the data-taking process, such as halting the accumulation of data in the correlator, may be used to achieve excision in the temporal domain. This method has been used successfully for impulsive and periodic signals [14]. The Arecibo Observatory implemented an airport radar blanking system that stops correlator processing in synch with the period of the radar pulse during a time window tailored to encompass the consequent radar artifacts from terrain and multi-path scattering. Lost data is counted as a loss of observing time when this variety of RFI occurs within an allocated RAS band.

Much research has been applied to the design of robust receivers with a high degree of linearity, so that harsh RFI environments do not affect them. Broadband observations are possible when receiver systems are sufficiently linear that no aliasing occurs, no inter-modulation products are generated, and no overloading occurs $[14,15,16]$.

\section{Pre-correlation}

\subsection{Antenna-based digital processing}

Real-time digital processing may be implemented as part of the IF processing of single-dish radio telescopes (RTs), and as part of the station processing and/or beamforming process for array instruments. This cost-effective method works well for impulsive (transient) RFI and requires fast data sampling as well as the availability of computing cycles at each of the stations $[17,18]$. The amount of data loss is determined by the transient nature of the RFI. Real-time, IF-based flagging and excising minimizes the data loss incurred by the flagging - excision method by only dealing with the RFI-infected time and frequency segments; this should not inflict collateral damage on 
neighboring time and frequency intervals. This is different from post-correlation processing, which is more vigorous as integrated data samples are used for baseline and antenna flagging and excising.

Thresholding in both the temporal and frequency domains may be applied when the RFI in sampled data is strong and identifiable, and the spectral occupancy of the RFI is relatively low. Thresholding was first used to remove RFI at the Ratan 600 telescope [19] and has been used many more times since. A recent successful application was at the Westerbork Synthesis Radio Telescope (WSRT), where $20 \mathrm{MHz}$ dual-polarization data from each of the fourteen telescopes was processed in real-time [20,21]. This thresholding method has also been applied to pulsar data prior to period folding $[22,23]$.

Sub-space filtering methods search for a particular signature in the RFI power component of data in order to identify and remove it. A particularly successful application is the search for cyclo-stationarity within data, which works well for digitally modulated RFI signals [24, 25].

Another form of sub-space excision exploits the probability distribution analysis of signals. Since the RFI contribution changes the power spectrum to a non-central (chi-square) distribution, as determined by its higher moments, it can be removed from data [1,26]. A similar approach is to use kurtosis (4th moment of the power spectrum) to identify and remove the RFI component. This has been used for single-dish solar observations [27, 28], and for (post-) correlation processing in a software correlation environment [29]. Median filtering and taking advantage of the median properties of a multi-feed system, also exploit the statistical properties of data and are effective in real-time RFI mitigation of spectral-line data [30, 31]].

Pre-correlation mitigation methods that involve the removal of data samples result in changes in the gain calibration of data. This requires accurate bookkeeping to determine the effect on the data and the associated data loss. Replacing affected data in the frequency (or time) domain with a fitted baseline only affects the rms of affected channels.

\subsection{Adaptive (temporal) noise cancellation}

Adaptive noise cancellation (ANC) is often used in communication and military technology [32]. The basic principle of temporal adaptive filtering is to make a FFT from the incoming data, perform an adaptation operation on the frequency bins, and then return to the frequency domain via an inverse FFT. This method, based on Wiener filtering, works for interfering signals with a significant INR, i.e. when the RFI dominates the system noise. The suppression of the interfering signal can be about equal to its instantaneous INR. Adaptive filters are effective when spectral information is unimportant, such as in pulsar [33] and continuum studies. This method has also been used effectively with multi-feed or focal plane arrays on single dishes (see next section). A variation on adaptive filtering is to subtract a reference data-channel from a signal data-channel using a copy of the RFI itself, by comparing on-source plus RFI and off-source plus RFI signals. In some existing telescope systems, the copy of the RFI is taken from an auxiliary reference antenna aimed at its source [34]. However, each distinct source of RFI then requires its own antenna.

Parametric estimation of known RFI signals and their subtraction from the data has been applied successfully for Glonass C/A satellite transmissions by exploiting their known modulation properties [35]: a signal cancellation of more than $20 \mathrm{~dB}$ was achieved without recourse to auxiliary antennas. A recent implementation of adaptive filtering techniques aims to remove the signature of the L3 transmission from a single GPS satellite at the Arecibo Observatory [36]. 


\subsection{Spatial filtering and null steering}

Every multiple-antenna array has sidelobes and nulls in its beam pattern that can be used to reduce signals from localized sources of RFI. In general, an adaptive system using a beam-forming algorithm requires a high INR and is limited to a small number of RFI targets to be tracked during an observation. The RFI sources also need to remain stable and predictable through an observation. Spatial filtering in beam-forming mode for a limited number of RFI sources generally does not degrade the image generated by the main beam.

Smart antenna techniques, using multiple sensors in radar and communication systems, are used to determine the direction-of-arrival and to implement beam-forming algorithms. Similarly, multiple-sensor, new-generation telescopes with a direct view of identified RFI sources (such as LOFAR and the Murchison Widefield Array) allow the beam-forming process to be optimized to include real-time, adaptive nulling and spatial filtering of these distinct RFI sources [37, 38, 39]. In a practical implementation, one hundred LOFAR antennas were used to generate two separate beams, while placing a permanent null at one position 15 degrees above the horizon [38]. Wellcalibrated, multi-sensor, phased arrays offer the possibility of steering a null to track a satellite while maintaining a high-gain beam on a target field [40], though the processing complexity increases rapidly if one is coping with a multi-satellite system.

For sparse arrays, with relatively long baselines, correlation may be performed first and the beams synthesized afterwards. Assuming the RFI sources are localized, their suppression is then achieved by processing short time intervals of the data stream, and applying complex weighting during image processing [41]. Computer simulations of post-correlation spatial filtering show that cleaning with an RFI-corrected beam can be effective [42].

Focal plane array (FPA) systems and multi-beam receivers provide new opportunities for spatial filtering, as each of the component feeds has an independent sky signal together with the common RFI signal $[43,44,45]$. In addition, one of the feeds in a multi-beam system can always be used as a reference antenna.

\section{At correlation}

As part of the correlation process, digitized data are generally integrated over time intervals ranging from the sampling time up to seconds, which significantly raises the INR. In consequence, persistent but weak RFI, that could not be treated in real-time, and weak (spectral) remnants of earlier mitigation operations become accessible for processing. On the other hand, significant peaks of a variable RFI signal may be reduced in strength by this integration. For array instruments, spatial filtering resulting from delay (fringe) tracking of a celestial source also reduces the strength of terrestrial RFI in cross-correlated data.

At this point in the data taking process, anti-coincidence protocols may be incorporated to identify the RFI components, as well as digital mitigation processing and the use of data from a reference antenna. New generation software correlators permit the integration of (kurtosis-based flagging) applications before and after FX (Fourier Transform before multiplication) correlation and stacking protocols [29]. Mitigation at different stages during processing is being implemented in LOFAR [46]. For single-dish instruments the correlation processing of (multiple) single bands 
may incorporate both thresholding or statistical methods and noise cancellation with a reference antenna.

Deploying digital processing and input from reference antennas during SW correlation is equivalent to the baseband pre-correlation processing outlined above. On the other hand, their implementation in conventional (existing) hardware backends requires the addition of both special hardware and software.

\section{Post-correlation - before or during imaging}

Traditional post-correlation processing consists of flagging and excising, which is time consuming and often done by hand [47]. Because this operation is performed on integrated and correlated data, the data loss resulting from flagging can be quite significant, the more as whole timeslots, whole baselines, and/or whole antennas may be flagged. This differs from antenna-based IF flagging or excising where small subsets are flagged, which inherently results in a smaller proportion of data loss overall.

On-line or off-line processing of (integrated) correlated data makes it possible to incorporate automated flagging and excision [48, 49, 50, 51], as more sophisticated statistical or sub-space processing (see section 6.1) can be implemented to remove the RFI component without much associated data loss.

Indeed, a reference antenna has been implemented at the post-correlation stage to remove the signal from a well-defined source using the available closure relations [52].

Array instruments employ fringe-stopping and delay-compensation techniques during observations to keep the fringe rate of the central observing position at zero during observations. As a result the stationary (terrestrial) and satellite RFI components in the data distinguish themselves by fringing faster than astronomical sources, i.e. at the fringe-stopping rate. This distinct (relative) motion allows the off-line identification and elimination of stationary RFI sources from both the correlated data and the image plane without causing data loss [53, 54, 55]. A code first applied at the GMRT is now incorporated into AIPS [56].

\section{Implementation at telescopes - strategy}

The data acquisition process of radio astronomy observatories is evolving to cope with the rapidly changing technological environment. The analog to digital conversions of signals now occurs as early as possible in the data handling scheme, which allows digital processing throughout most of the data chain. Increased capabilities allow the processing of larger bandwidth data, with higher time-resolution and higher frequency $(<\mathrm{kHz})$ resolution.

Many current backends do not allow the implementation of mitigation at early stages of the data handling chain without incurring (severe) hardware modifications. By contrast, newgeneration backends and software correlation facilitate such schemes at different stages of the processing.

Since every mitigation method requires a definite INR threshold for its operation, removal of most of the RFI requires a layered application of methods to exploit the progressive integration of the data and its increasing INR. While no method can remove RFI below the noise floor it 
encounters, subsequent mitigation steps may remove remnants of the mitigated RFI, as well as weak RFI that is only apparent after integration.

The implementation of auxiliary antennas for array instruments depends on the possibility of incorporating their output into the processing system, (most particularly) at the correlator. Directed reference antennas generally cope with particular RFI sources and are less effective in a complicated environment.

Human intervention in the RFI mitigation process plays an important role in practical operations. Thus real-time on-line processing that is adaptable to a variety of RFI signatures may be preferred to the restrictive use of reference antennas and/or spatial filtering for known and fixed transmitters. This is likely to be the case until some artificial intelligence controller can be invoked to guide and dictate the RFI mitigation scheme.

Interferometers are less vulnerable to RFI. Fringe-stopping and decorrelation by delay compensation provide for its natural suppression on the longer baselines. However, strong RFI still adds to the system noise, and still affects the calibration and the complex visibilities of a station. VLBI stations and distributed sensor networks can implement mitigation at every individual station to reduce the impact of local RFI on the whole system.

To correctly calibrate a system, accurate bookkeeping is required for all affected data in order to obtain the correct weights for later self-calibration, cleaning and imaging procedures.

Future mitigation implementations need to consider more sophisticated methods than the simple (kurtosis or other) RFI flagging and excising algorithms that are generally current at this time. The use of statistical methods using higher moments opens the possibility of removing RFI components without affecting the rest of the data, and there are methods that allow partial restoration of data that reduce data loss. Adaptive filtering of spread-spectrum systems may become possible when their digital keying schemes are known.

\section{Conclusions}

Both on-line and off-line data processing has been successful in mitigating the RFI environment of radio astronomy observatories. While there is an increasing variety of successful mitigation options, the choice of method depends strongly on the RFI characteristics, the type of radio telescope, and the type of observation. In particular, on-line real-time data-processing may be preferred in a variable RFI environment, while special measures such as reference antennas and spatial filtering may be preferred for known and fixed sources of RFI. In addition to these factors, the absence of human involvement may also render automated on-line processing a more attractive option.

No universal method exists for mitigating RFI in astronomical data and no method can identify or remove RFI within the noise of the system. The effective suppression of RFI depends on the INR and its temporal and spectral characteristics. A quantitative evaluation of the method used is not always possible because mitigation algorithms are generally non-linear processes that also affect the noise characteristics and the gain calibration. The toxicity of the method used, i.e. the negative effect of its invocation on data by the deployed method, and the amount of data loss resulting from the method are other factors that guide the evaluation of the choice of method.

Multiple methods need to be applied to deal with a more general RFI environment. Because RFI characteristics change after each mitigation step and with increasing integration of the data, the 
cumulative effect of RFI mitigation at subsequent stages is not a linear sum of what each method can do, but rather the sum of what is practical and possible at each step.

The cost of computing hardware capability and of digitizing components at radio astronomy observatories is rapidly changing. Both upgrades of existing facilities and the introduction of newly constructed instruments provide opportunities for implementing and automating RFI mitigation algorithms. These capabilities also permit increased bandwidth, higher time resolution, and higher spectral resolution. The resulting, increasingly large data volumes will force the introduction of automated data reduction pipelines.

During recent years, spectrum management and on-line RFI mitigation have not been given the attention they deserve. As RFI could be flagged and excised from the data and the (mostly allocated) observing bands were relatively clean, the traditional user community learned to live with and accept the presence of RFI. Few observing bands suffered significant loss. However, without national and international spectrum management efforts the current state of the RAS allocated bands would have been far worse. The use by radio astronomy stations of much broader operating bandwidths that also cover allocations of other services will demand continued spectrum management efforts. In addition, future data volumes are likely to force the acceptance of automated RFI mitigation at radio observatories.

New telecommunication and broadcasting technologies are reaching the market place, many in the form of unlicensed mobile devices. Since their ever-changing locations are impossible to control, they will rapidly affect observatory operations. Algorithmic research is needed to eliminate their signals from astronomical data. In particular, spread spectrum (ultra-wide band) devices will pose problems for passive services, as their digital modulation schemes do not respect the boundaries of spectrum allocations. Current estimates suggest that the number of transmitting devices used by each person is set to increase dramatically and many of these will rely on dynamic spectrum access.

The discovery space for radio astronomy is determined to a significant degree by the technical characteristics of the observing system and by limiting factors such as the RFI environment. While new generation telescopes are located at the most pristine possible sites, existing facilities must coexist with their local conditions. In order to prevent RFI becoming the limiting factor for existing facilities, spectrum management, both internal and external, has to be accorded a very high priority. Both observatory management and astronomers should regard RFI issues as critical.

\section{References}

[1] Fridman, P. A. \& Baan, W. A. RFI mitigation methods in radio astronomy, A\&A, 378, 327, 2001

[2] International Telecommunication Union, Report ITU-R RA.2126, Techniques for mitigation of radio frequency interference in radio astronomy, Geneva, 2007

[3] Kesteven, M. The current status of RFI mitigation in radioastronomy, 2009, IAU

[4] Ellingson, S. W., Introduction to special section on mitigation of radio frequency interference in radio astronomy, Radio Science, vol. 40, 5, RS5S01, 2005

[5] Briggs, F.H. \& Kocz, J., Overview of Technical Approaches to Radio Frequency Mitigation, Radio Science, vol. 40, 5, RS5S02, 2005. 
[6] Thompson, A.R., The response of a radio astronomy synthesis array to interfering signals, IEEE Transactions on Antennas and Propagation, vol. AP-30, pp. 450Đ456, 1982.

[7] Cloudsat satellite radar system, www.cloudsat.atmos.colostate.edu/

[8] International Telecommunication Union, ITU-R Handbook on Radio Astronomy, Geneva, Switzerland, 2003.

[9] International Telecommunication Union, Recommendation ITU-R RA.769-2, Protection criteria used for radio astronomical measurements, Geneva, Switzerland, 2003, and Recommendation ITU-R RA.1513-1, Levels of data loss to radio astronomy observations and percentage-of-time criteria resulting from degradation by interference for frequency bands allocated to the radio astronomy on a primary basis, Geneva, Switzerland, 2003

[10] Cohen, J., Spoelstra, T., Ambrosini, R. \& van Diel, W., CRAF Handbook for Radio Astronomy (Third Edition), European Science Foundation, Strasbourg, France, 2005 and www.craf.eu

[11] National Radio Quiet Zone, www.gb.nrao.edu/nrqz/nrqz.html

[12] Puerto Rico Coordination Zone, www.naic.edu/techinfo/prcz/prczinfo.htm

[13] Rogers, A.E.E., Pratap, P., Carter, J.C., \& Diaz, M. Radiofrequency interference shielding and mitigation techniques for a sensitive search for the $327 \mathrm{MHz}$ line of Deuterium, Radio Science, 40, no. 5,2005

[14] Weber, R., Faye, C., Biraud, F., \& Dansou, J., Spectral detector for interference time blanking using quantized correlator, A\&AS, 126, 161, 1997

[15] Clerc, V., Weber, R., Denis, L. \& Rosolen, C., High Performance Receiver for RFI Mitigation in Radio Astronomy: Application at Decameter Wavelengths, EUSPICO’02, Toulouse, France, 2002, and Robust Receiver for RFI Mitigation in Radio Astronomy, Proceedings of the XXVII General Assembly, International Union of Radio Science, Maastricht, The Netherlands, 2002.

[16] Tuccari, G., Caddemi, A., Nicotra, G. \& Consoli, F., Cryogenic Filters for RFI Mitigation in Radioastronomy, Proc. 7th European VLBI Network Symposium. Toledo, Spain, 2004.

[17] Niamsuwan, N., Johnson, J.T. \& Ellingson, S.W., Examination of a simple pulse-blanking technique for radio frequency interference mitigation, Radio Science, vol. 40, no. 5, 2005

[18] Ellingson, S.W. \& Hampson, G.A., Mitigation of Radar Interference in L-Band Radio Astronomy, Astrophys. J. Suppl. Ser, 147, 167, 2003.

[19] Berlin, A. B. \& Fridman P., Real-Time Radiometric Data Processing against Electromagnetic Pollution, Proc. XXV URSI GA (URSI Gent), 750, 1996

[20] Baan, W.A., Fridman, P.A., \& Millenaar, R.P., Radio frequency interference mitigation at the Westerbork Synthesis Radio Telecope: algorithms, test observations, and system implementation, AJ $128,933,2004$

[21] Baan, W.A., Fridman, P.A., Millenaar, R.P. \& Roy, S., The WSRT Interference Mitigation System lessons learned, PoS(RFI2010)024, 2010

[22] Fridman, P., Statistically Stable Estimates of Variance in Radio-Astronomy Observations as Tools for Radio-Frequency Interference Mitigation, AJ, 135, 1810, 2010

[23] Fridman, P.F. Robust Correlators, A\&A 502, 401, 2009.

[24] Weber, R., Zarka, P., Ryabov, V.B, et al., Data Preprocesing for Decametre Wavelength Exoplanet Detection: an Example of Cyclostationary RFI Detector, EUSPICO'07, Poznan, 2007 
[25] Feliachi, R., Weber, R. \& Boonstra, A-J., Cyclic Spatial Filtering in Radio Astronomy: Application to LOFAR Data, EUSPICO'09, Glasgow, UK, 2009, and Cyclostationarity for phased array radio telescopes, PoS(RFI2010)033, 2010

[26] Fridman, P. A., RFI excision using a higher order statistics analysis of the power spectrum, A\&A, $368,369,2001$

[27] Nita, G.M., Gary, D.E., Liu, Z., Hurford, G.J. \& White, S.M., Radio Frequency Interference Excision Using Spectral Domain Statistic, PASP, 119, 805

[28] Gary, D.E., Liu, Z. \& Nita, D.M, A Wideband Spectrometer with RFI Detection, PASP, 122, 560, 2010

[29] Deller, A. Software correlators as testbeds for RFI algorithms, PoS(RFI2010)035, 2010

[30] Kalberla, P. RFI mitigation of the Parkes Galactic All-Sky Survey (GASS), PoS(RFI2010)038, 2010

[31] Flöer, L., Winkel, B. \& Kerp, J., RFI Motigation for the Effelsberg-Bonn HI survey (EBHIS), PoS(RFI2010)042, 2010

[32] Haykin, S. 2001, Adaptive Filter Theory (4th ed.; Prentice Hall)

[33] Kesteven, M., Hobbs, G., Clement, R., Dawson, B. , Manchester, R. \& Uppal, T., Adaptive Filters Revisited - RFI Mitigation in Pulsar Observations, Radio Science, vol. 40, no. 5, RS5S06, 2005.

[34] Barnbaum, C., \& Bradley, R. F. A new approach to interference excision in radio astronomy: Real-time adaptive filtering, AJ, 116, 2598, 1998

[35] Ellingson, S. W., Bunton, J. D., \& Bell, J. F., Removal of the GLONASS C/A Signal from OH Spectral Line Observations Using a Parametric Modeling Technique, ApJS, 135, 87 Glonass C/A

[36] Nigra, L., Lewis, B.M., Edgar, C., et al. A turn-key concept for active cancellation of the Global Positioning System L3 signal, PoS(RFI2010)025, 2010

[37] Ardenne van, A., Smolders, B., \& Hampson, G., Active adaptive antennas for radio astronomy: results from the $R \& D$ program on the Square Kilometer Array, Proc. SPIE, 4015, 420, 2000

[38] Bregman, J. D., Concept design for a low-frequency array, Proc. SPIE, 4015, 19, 2000

[39] Leshem, A., van der Veen, A. J., \& Boonstra, A.-J., Multichannel interference mitigation techniques in radio astronomy, $\mathrm{ApJ}, 131,355,2000$

[40] Fridman, P.A., RFI mitigation with phase-only adaptive beamforming, Radio Science, 40, n.2, 2005

[41] Harp, G.R., The ATA digital processing requirements are driven by rfi concerns, Radio Science, vol. 40, no. 5, RS5S18, 2005.

[42] Leshem, A. \& van der Veen, A.J., Radio astronomical imaging in the presence of strong radio interference, IEEE Transactions on Information Technology, vol. IT-46, no. 5, pp. 1730, 2000.

[43] Boonstra, A.J. \& van der Tol, S., Spatial filtering of interfering signals at the initial low frequency array (LOFAR) phased array test station, Radio Science, p. 40, RS5S09, 2005

[44] Hansen, C. K., Warnick, K. F., Jeffs, B. D., Fisher, J. R. and Bradley, R., Interference mitigation using a focal plane array, Radio Science, p. 40, RS5S15, 2005

[45] Kocz, J., Briggs, F.H. \& Reynolds, J., Radio frequency interference removal through the application of spatial filtering techniques on the Parkes multibeam receiver, AJ, submitted, 2010.

[46] Bentum, M., Boonstra, A-J., Millenaar, R.P. \& Gunst, A., Implementation of LOFAR RFI mitigation strategy, URSI General Assembly 2008, Chicago, USA. 
[47] Lane, W.H., Cohen, A.S., Kassim, N.E., et al., Postcorrelation radio frequency interference excision at low frequencies, Radio Science, vol. 40, no. 5, RS5S05, 2005.

[48] Middelberg, E., Automated Editing of Radio Interferometer Data with Pieflag, PASP, vol. 23, 2006.

[49] Offringa, A. R., et al., Post-correlation radio frequency interference classification methods, 2010, MNRAS, 405, 155, and Offringa, A. R., de Bruyn, A. G., Zaroubi, S. \& Biehl, M., A LOFAR RFI detection pipeline and its first results, 2010, arXiv:astro-ph/1007.2089

[50] Keating, G., Barott, W.C. \& Wright, M., Automated calibration and imaging with the Allen Telescope Array, Proc. SPIE, 7740, 39, 2010

[51] Sirothia, S.K. et al., Deep low-frequency observations with the Giant Metrewave Radio Telescope: a search for relic radio emissionMNRAS, 392, 1403, 2009, and 325-MHz observations of the ELAIS-N1 field using the Giant Metrewave Radio Telescope, MNRAS, 395, 269, 2009

[52] Briggs, F. H., Bell, J. F., \& Kesteven, M. J., Removing radio interference from contaminated astronomical spectra using an independent reference signal and closure relations, AJ, 120, 3351, 2000

[53] Wijnholds, S.J., Bregman, J.D. \& Boonstra, A-J., Sky noise limited snapshot imaging in the presence of rfi with LOFAR'ss initial test station, in RFI2004 Penticton, Canada, 2004.

[54] Cornwell,T.J., Perley, R.A., Golap, K. \& Bhatnagar, S., RFI excision in synthesis imaging without a reference signal, EVLA memo series 86, 2004.

[55] Athreya, R., A New Approach to Mitigation of Radio Frequency Interference in Interferometric Data, 2009, ApJ 696, 885

[56] Kogan, L. \& Owen, F., RFI Mitigation in AIPS. The New Task UVRFI, PoS(RFI2010)037, 2010 